\title{
Novel Approach in the Construction of Bioethanol-Producing Saccharomyces cerevisiae Hybrids ${ }^{\S}$
}

\author{
Anamarija Štafa', Bojan \\ Žunar', Andrea Pranklin', \\ Antonio Zandona', \\ Marina Svetec Miklenić', \\ Božidar Šantek² and \\ Ivan Krešimir Svetec ${ }^{1 *}$
}

'University of Zagreb, Faculty of Food Technology and Biotechnology, Department of Biochemical Engineering, Laboratory for Biology and Microbial Genetics, Kršnjavoga 25, 10000 Zagreb, Croatia

2University of Zagreb, Faculty of Food Technology and Biotechnology,

Department of Biochemical Engineering, Laboratory for Biochemical Engineering, Industrial Microbiology and Malting and Brewing Technology, Kačićeva 28, 10000 Zagreb, Croatia

Received: 25 January 2018 Accepted: 10 December 2018

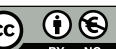

\begin{abstract}
SUMMARY
Bioethanol production from lignocellulosic hydrolysates requires a producer strain that tolerates both the presence of growth and fermentation inhibitors and high ethanol concentrations. Therefore, we constructed heterozygous intraspecies hybrid diploids of Saccharomyces cerevisiae by crossing two natural S. cerevisiae isolates, YIIc17_E5 and UWOPS87-2421, a good ethanol producer found in wine and a strain from the flower of the cactus Opuntia megacantha resistant to inhibitors found in lignocellulosic hydrolysates, respectively. Hybrids grew faster than parental strains in the absence and in the presence of acetic and levulinic acids and 2-furaldehyde, inhibitors frequently found in lignocellulosic hydrolysates, and the overexpression of YAP1 gene increased their survival. Furthermore, although originating from the same parental strains, hybrids displayed different fermentative potential in a $\mathrm{CO}_{2}$ production test, suggesting genetic variability that could be used for further selection of desirable traits. Therefore, our results suggest that the construction of intraspecies hybrids coupled with the use of genetic engineering techniques is a promising approach for improvement or development of new biotechnologically relevant strains of $S$. cerevisiae. Moreover, it was found that the success of gene targeting (gene targeting fidelity) in natural S. cerevisiae isolates (Yllc17_E5a and UWOPS87-2421a) was strikingly lower than in laboratory strains and the most frequent off-targeting event was targeted chromosome duplication.
\end{abstract}

Key words: yeast Saccharomyces cerevisiae, intraspecies hybrids, lignocellulosic hydrolysates, growth and fermentation inhibitors, gene targeting

\section{INTRODUCTION}

The requirement for the fuel is constantly increasing and the production of biofuels (bioethanol, biodiesel and biobutanol) from renewable sources has become more frequent in the last several decades. Biofuels are usually classified depending on the source of material used for production (1). Biofuels of the first generation are made by fermentation of raw starch-containing material that can be also used for human consumption, resulting in both expensive and unethical production. Biofuels of the second generation are made using renewable lignocellulosic waste, while third generation biofuels are made from algae and microbial biomass (1).

Although lignocellulosic waste consists of cellulose (35-50\%), hemicellulose (20-35\%) and lignin (5-30\%), its exact composition depends on the origin of the material (2). Cellulose is a linear polymer of glucose, hemicellulose consists of D-xylose, L-arabinose, D-glucose, D-galactose and D-glucuronic acid, while lignin consists of $p$-coumaryl, conypheryl and synapyl alcohol (3). Since microorganisms are not able to ferment raw lignocellulosic material directly, it is first pretreated to release compounds that can be further hydrolysed and then used by yeasts for fermentation. Although there are different methods of pretreatment (chemical, physical, physicochemical and enzymatic), the choice of method is still one of the main challenges resulting in hydrolysates whose chemical composition depends both on the type of starting material and the type of pretreatment. The pretreatment does not result in a complete degradation of a starting material releasing 
fermentable sugars, it also generates different compounds that act as growth and fermentation inhibitors. Hydrolysis of cellulose releases glucose, while hydrolysis of hemicellulose releases xylose, arabinose, galactose and glucose. Further degradation of xylose results in the formation of 2-furaldehyde (furfural), and the degradation of mannose, galactose and glucose releases 5-hydroxymetylfurfural (HMF). Acetyl groups of hemicellulose form acetic acid, while formic and levulinic acid are released during the pretreatment of 5-hydroxymethylfurfural and 2-furaldehyde. Although aliphatic acids can be used by yeast as a carbon source if their concentration is below $100 \mathrm{mM}$ (4), when present in higher concentrations they enter cells, dissociate and decrease intracellular $\mathrm{pH}$ resulting in the increase of a doubling time (3). Phenols released during the pretreatment have different effects on yeast because their inhibitory activity depends on functional side groups and the number of unsaturated bonds between carbon atoms (5). The 2-furaldehyde decreases both the growth rate and ethanol yield, but its inhibitory effect also depends on cell density, conditions of cultivation and aeration (6). Comparative analysis of four different lignocellulose hydrolysates showed that 2-furaldehyde was the most toxic compound (7). Ethanol is the main product of fermentation and it changes the expression of genes involved in ionic homeostasis, trehalose synthesis and antioxidant defence, and of those encoding heat shock proteins (8). Moreover, each inhibitor alone negatively influences yeast cell growth but the combination of acetic acid, aldehydes and furan compounds or acetic acid and 2-furaldehyde synergistically decreases the growth rate $(9,10)$.

Since the fermentation inhibitors are released regardless of the type of pretreatment, there have been different approaches to increase the resistance of producer strains. These approaches range from the use of different industrial strains (11) to the adaptation of the strains to the presence of inhibitors $(12,13)$ or by different genetic modifications of the producer strains. Both endogenous yeast genes and genes from different species have been expressed in S. cerevisiae in order to increase the resistance of producer strains. Expression of LCC2 gene from Trametes versicolor in S. cerevisiae allowed simultaneous detoxification and fermentation (14), while overexpression of genes from pentose phosphate pathway conferred tolerance to weak acids (15). Alriksson et al. (16) constructed strains that overexpressed endogenous ATR1, FLR1 or YAP1 gene, involved in multidrug resistance or stress tolerance, and the most positive effect was observed after overexpression of YAP1 gene that encodes transcription factor involved in stress response. Ask et al. (17) overexpressed genes involved in glutathione synthesis resulting in the resistance to inhibitors and an increase in ethanol production.

In this work, we constructed intraspecies hybrids of $S$. cerevisiae strains by mating haploids of two natural isolates having different properties desirable for bioethanol producer, such as high ethanol production and resistance to several growth and fermentation inhibitors. The constructed hybrid diploids survived better than both parental haploids and diploids in the presence of acetic acid, levulinic acid and 2-furaldehyde. In order to further increase the survival of constructed strains, the influence of the overexpression of ATR1, FLR1, YAP1 and GSH1 genes was tested. Although there was a difference in the increase of the survival of different strains, the overexpression of YAP1 gene had a more positive overall effect than the overexpression of ATR1 gene, whereas the overexpression of FLR1 or GSH1 gene did not have any significant influence. Moreover, constructed intraspecies hybrids displayed genetic variability that could be used for further selection of desirable traits. Therefore, construction of intraspecies hybrids is a promising approach for an improvement or development of new biotechnologically relevant $S$. cerevisiae strains.

\section{MATERIALS AND METHODS}

\section{Plasmids}

Plasmids used in this study are listed in Table S1. The plasmid pRED150 (18) was used to change the genotype of parental YIIc17_E5a and UWOPS87-2421a strains from ADE2 ura3 (phenotype Ade ${ }^{+} \mathrm{Ura}^{-}$) to ade2 URA3 (phenotype Ade- Ura ${ }^{+}$) by plasmid integration in ADE2 locus (Fig. 1). This plasmid contains a $150 \mathrm{bp}$ long perfect palindrome which strikingly enhances plasmid pop-out (19) and is used to restore original ADE2 ura3 genotype in diploids constructed by mating (Fig. 2 $(19,20))$. Plasmid pRED150 is constructed from pAB9-150 (19) by replacing $C Y C 1$ region with 1.1-kb central part of the $A D E 2$ gene (from EcoRV to Dral cut site), whereas the approach for a perfect palindrome construction is described by Svetec et al. (21). All other plasmids used in this work were constructed by PCR amplification, using Q5 Polymerase (New England Biolabs (NEB), Ipswich, MA, USA), of genes from UWOPS87-2421 genome and were cloned in MCS of pSP-G2 (22) or PSP-AC (complete list of primers with restriction sites is listed in Table S2. Standard media and procedures were used for the cultivation of the Escherichia coli strains (DH5a and XL1blue) and all DNA manipulations (23).

\section{Yeast strains}

Saccharomyces cerevisiae strains used in this study are listed in Table 1 (24,25), while schematic representation of the construction of all strains is shown in Fig. $2(19,20)$. Throughout the work, standard methods for yeast cultivation and manipulation were used (26). Strains 2421REDa and E5REDa were constructed by integration of pRED150 in ADE2 locus of UWOPS87-2421a and YIIc17_E5a, respectively (Fig. 1). Transformants obtained by transformation with pRED150 were patched on synthetic complete (SC) without uracil (SC-Ura) plates and yeast extract-peptone-dextrose (YPD) medium and replica plated to SC-Ade plates to verify ade2 URA3 genotype. Ade- Ura ${ }^{+}$transformants were analysed by Southern 
a)

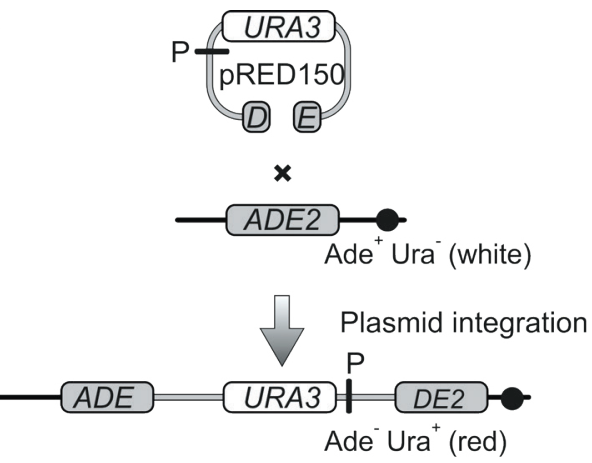

b)

$\begin{array}{lllllllllllll}1 & 2 & 3 & 4 & 5 & 6 & 7 & 8 & 9 & 10 & 11 & 12 & M\end{array}$

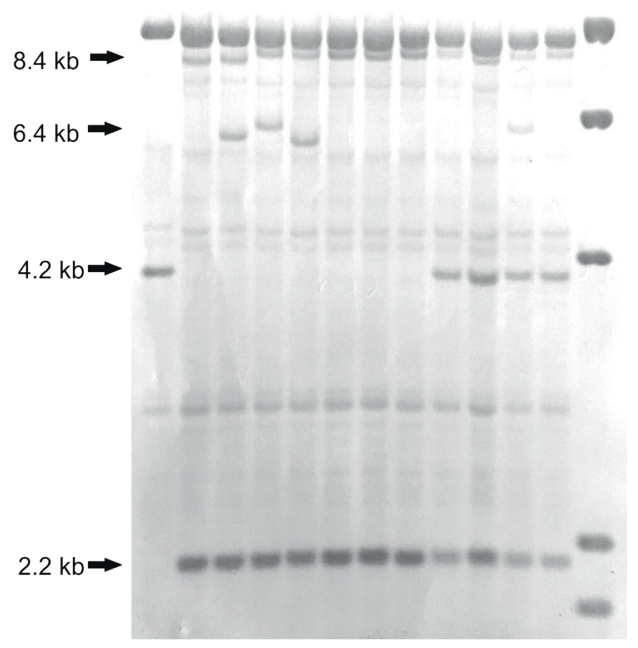

c) Lane 1 - untransformed strain

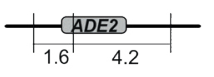

Lanes 2, 6, 7, 8 - plasmid integration

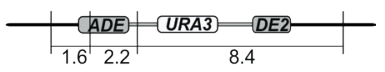

Lanes 3, 4, 5 - multiple plasmid integration

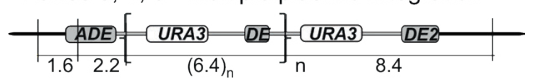

Lanes 9, 10,12- heteroallelic transformants

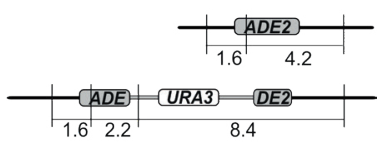

Lane 11 - heteroallelic transformant with multiple plasmid integration

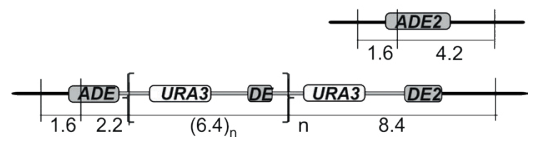

Fig. 1. Construction of 2421_REDa and E5_REDa strains by transformation of UWOPS87-2421a and Yllc17_E5a strains, respectively, by pRED150 plasmid: a) schematic representation of the plasmid integration assay, $\mathrm{P}$ denotes $150 \mathrm{bp}$ palindrome, b) typical results of the molecular analysis of transformants, and c) schematic representation of the transformation events observed during Southern blotting. To allow better separation of longer DNA fragments, $1.6-\mathrm{kb}$ band that would have been hybridized with ADE2 gene in all samples was allowed to exit the gel. Genomic DNA of the transformants was cut with Asel

blotting to confirm plasmid integration in ADE2 region. Two Ade-Ura ${ }^{+}$transformants (strains 2421REDa and E5REDa), containing a single pRED150 molecule integrated in ade2 locus, were mated with haploids (UWOPS87-2421a and YIIc17_E5a) with $\mathrm{Ade}^{+}$Ura phenotype to construct $\mathrm{Ade}^{+} \mathrm{Ura}^{+}$diploid heterozygous hybrids $(\mathrm{H} 1, \mathrm{H} 2, \mathrm{H} 3$ and $\mathrm{H} 4)$ and homozygous controls (2421_C1 and E5_C2) (Fig. $2(19,20))$. Hybrids H1 and $\mathrm{H} 4$ were isolated as single colonies from a cross of a-mating 2421REDa and a-mating YIIc17_E5a, while H2 and H3 were taken as single colonies from a cross of a-mating E5REDa and a-mating UWOPS87-2421a. In order to restore the genotype of parental strains ( $\mathrm{Ade}^{+} \mathrm{Ura}^{-}$), six constructed $\mathrm{Ade}^{+} \mathrm{Ura}^{+}$diploids (H1-H4, 2421_C1 and E5_C2) were grown in complete YPD medium to allow for pop-out of the plasmid pRED150 integrated in ade2 region, resulting in ADE2 ura3 genotype (Fig. $2(19,20))$.
Karyotype of parental a-mating type haploid strains (UWOPS87-2421a and YIIc17_E5a) was confirmed by quantitative polymerase chain reaction ( $\mathrm{PPCR}$ ) copy number analysis for all 16 yeast chromosomes as described previously (27).

\section{Yeast transformation and molecular analysis of transformants by Southern blotting}

Lithium acetate transformation was done as described previously (28) but since it was observed that the efficiency of transformation in natural isolates was lower than in commonly used laboratory strains, cells were first allowed to recover for $30 \mathrm{~min}$ in rich YPD medium and then plated on selective SC-Ura plates. Isolation of the genomic DNA was performed as described previously (29) and molecular analysis of transformants was done by Southern blotting (30) using dioxigenin (DIG)-labelled ADE2 gene (Roche, Darmstadt, 
a)

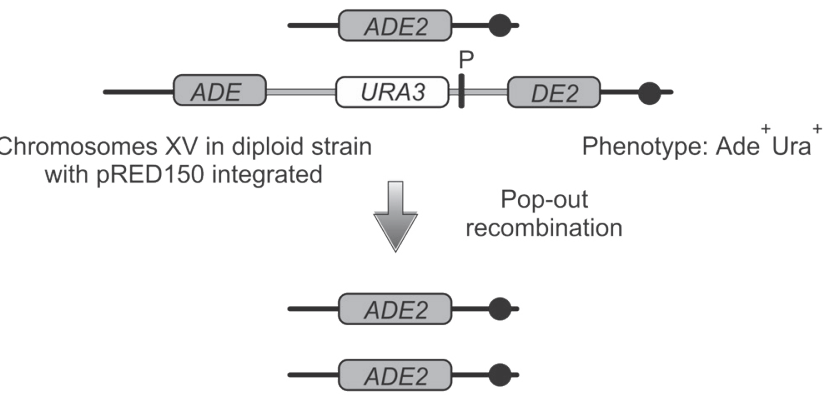

Chromosomes $\mathrm{XV}$ in diploid strain

Phenotype: $\mathrm{Ade}^{+}$Ura after pop-out

b)

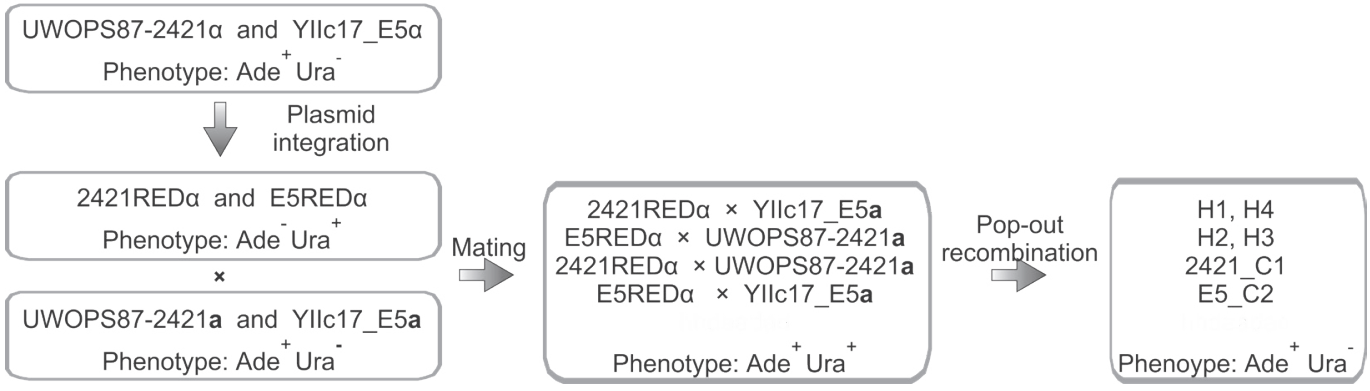

Fig. 2. Pop-out (loss) of the pRED150 plasmid and construction of hybrid and control diploid strains: a) plasmid pRED150 was previously integrated in the ade2 locus on chromosome XV in order to facilitate diploid construction. P represents 150 bp palindrome that stimulates pop-out recombination resulting in the loss of the plasmid containing URA3 gene (19) and restoration of ADE2 gene; pop-out recombinants were selected on 5-FOA plates (20), and b) strains UWOPS87-2421a and Yllc17_E5a were transformed with plasmid pRED150 (see Fig. 1) to construct 2421RADa and E5REDa strains which were mated with UWOPS87-2421a and Yllc17_E5a strains in order to construct Ade ${ }^{+}$Ura ${ }^{+}$diploids. Afterwards, the constructed diploids were grown under non-selective conditions to allow pop-out (loss) of pRED150 in order to construct Ade ${ }^{+} \mathrm{Ura}^{-}$hybrids ( $\mathrm{H} 1$, $\mathrm{H} 2, \mathrm{H} 3$ and H4) and homozygous controls (2421_C1 and E5_C2)

Germany). Statistical analysis of the spectra of transformation events was done using two-tailed Pearson's chi-squared test $\left(X^{2}\right)$.

\section{Semi-quantitative test of fermentative activity}

Assessment of fermentation ability was done using YPD and SC plates containing $130.0 \mathrm{mg} / \mathrm{L}$ bromothymol blue (Kemika, Zagreb, Croatia, BTB plates $(31,32))$. Yeast cultures were grown to the stationary phase in YPD or SC medium, decimal dilutions were prepared and spotted on BTB plates. Additionally, $100 \mu \mathrm{L}$ of sixth decimal dilution of yeast cultures were plated on BTB plates to allow for the growth of single colonies.

\section{Analysis of the growth in the presence of inhibitors}

Growth in the presence of acetic and levulinic acids and ethanol (Carlo Erba, Barcelona, Spain), and 2-furaldehyde (Acros Organics, New Jersey, USA) was determined in liquid media and/or on solid plates. For the analysis of growth in liquid media, strains were grown in YPD to the stationary phase, diluted in YPD to $A_{600 \mathrm{~nm}}=0.01$, and $4-\mathrm{mL}$ aliquots were put in test tubes containing a specific volume of $1 \mathrm{M}$ inhibitor stock.
If the volume of the inhibitor stock was bigger than $0.5 \mathrm{~mL}$, additional control samples that contained the same volume of water were prepared to correct for the medium dilution. Cultures were grown in an orbital shaker at $150 \mathrm{rpm}$ and 28 ${ }^{\circ} \mathrm{C}$, and all absorbance values were measured at time points $0,3,6,12$ and $24 \mathrm{~h}$. For each strain/inhibitor pair, two to four independent experiments were performed and all absorbance measurements were done by mixing the culture with $0.1 \mathrm{M}$ sodium citrate in 1:1 volume ratio to disperse any floccules that might have formed during growth. In all experiments in liquid media, yeast strain CEN.PK2 was used as an additional control.

To determine growth inhibition on solid media, complex complete (YPD), synthetic complete (SC) or synthetic complete without uracil (SC-Ura) plates with the addition of inhibitors were prepared. Strain precultures were grown to stationary phase in YPD or SC-Ura medium and the first decimal dilution was prepared in $0.1 \mathrm{M}$ sodium citrate to disperse all formed floccules. All other serial dilutions were prepared using sterile water and $5 \mu \mathrm{L}$ of each serial dilution were spotted on a plate. Plates were incubated for 4 days at $28^{\circ} \mathrm{C}$ and colonies were counted on the second and fourth day to calculate the percentage of survival. 
Table 1. Yeast strains used in this study

\begin{tabular}{|c|c|c|}
\hline Strain name & Genotype & Reference \\
\hline \multicolumn{3}{|l|}{ Parental haploid strain (Ade ${ }^{+}$Ura') } \\
\hline UWOPS87-2421a (NCYC 3582) & MATa ura3::KanMX ho::HygMX & \multirow{4}{*}{$(24)$} \\
\hline UWOPS87-2421a (NCYC 3609) & MATa ura3::KanMX ho::HygMX & \\
\hline YIIc17_E5a* (NCYC 3586) & MATa ura3::KanMX ho::HygMX & \\
\hline Yllc17_E5a* (NCYC 3612) & MATa ura3::KanMX ho::HygMX & \\
\hline \multicolumn{3}{|c|}{ Parental haploid strain transformed with pRED150 (Ade- Ura ${ }^{+}$) } \\
\hline 2421REDa & MATa ura3::KanMX ho::HygMX ade2::pRED150 & \multirow{2}{*}{ This study } \\
\hline E5REDa & MATa ura3:::KanMX ho::HygMX ade2::pRED150 & \\
\hline \multicolumn{3}{|l|}{ Heterozygous hybrid diploid strain (Ade ${ }^{+}$Ura') } \\
\hline \multicolumn{3}{|l|}{ H1 (2421REDa $\times$ Yllc17_E5a)** } \\
\hline H4 (2421REDa $\times$ Yllc17_E5a) $)^{* *}$ & \multirow{3}{*}{ MATa/a ura3::KanMX/ura3::KanMX ho::HygMX/ho::HygMX } & \multirow{3}{*}{ This study } \\
\hline H2 (E5REDa $\times$ UWOPS87-2421a) ${ }^{* * *}$ & & \\
\hline H3 (E5REDa $\times$ UWOPS87-2421a)*** & & \\
\hline \multicolumn{3}{|l|}{ Control homozygous diploid strain (Ade ${ }^{+}$Ura-) } \\
\hline 2421_C1 (2421REDa × UWOPS87-2421a) & \multirow{2}{*}{ MATa/a ura3::KanMX/ura3::KanMX ho::HygMX/ho::HygMX } & \multirow{2}{*}{ This study } \\
\hline E5_C2 (E5REDa $\times$ Yllc17_E5a) & & \\
\hline
\end{tabular}

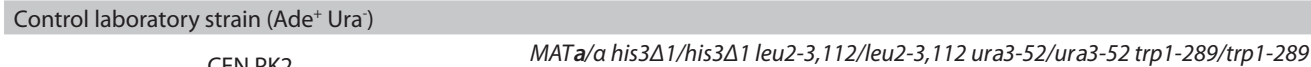

MATa/a his3D1/his3D1 leu2-3,112/leu2-3, 112 ura3-52/ura3-52 trp1-289/trp1-289
MAL2-8c/MAL2-8c SUC2/SUC2

Diploid strain transformed with plasmids PSP-G2

$$
\begin{gathered}
\text { H1-pSP } \\
\text { H2-pSP } \\
\text { H3-pSP } \\
\text { H4-pSP } \\
2421 \text { 1_1-pSP } \\
\text { E5_C2-pSP }
\end{gathered}
$$

$$
\begin{gathered}
\text { H1-ATR } \\
\text { H2-ATR1 } \\
\text { H3-ATR1 } \\
\text { H4-ATR1 } \\
\text { 2421_C1-ATR1 } \\
\text { E5_C2-ATR1 }
\end{gathered}
$$$$
\text { MATa/a ura3::KanMX/ura3::KanMX ho::HygMX/ho::HygMX pSP-ATR1 }
$$

Diploid strain transformed with plasmids PSP-YAP1
H1-YAP1
H2-YAP1
H3-YAP1
H4-YAP1
2421_C1-YAP1
E5_C2-YAP1

MATa/a ura3::KanMX/ura3::KanMX ho::HygMX/ho::HygMX pSP-YAP1

This study

Diploid strain transformed with plasmids PSP-FLR1

H1-FLR1

H2-FLR1

H3-FLR1

H4-FLR1

MATa/a ura3::KanMX/ura3::KanMX ho::HygMX/ho::HygMX pSP-FLR1

This study

2421_C1-FLR1

E5_C2-FLR1

Diploid strain transformed with plasmids PSP-AC
$\mathrm{H} 1-\mathrm{AC}$
$\mathrm{H} 2-\mathrm{AC}$
H3-AC
$\mathrm{H} 4-\mathrm{AC}$
2421_C1-AC
E5_C2-AC

MATa/a ura3::KanMX/ura3::KanMX ho::HygMX/ho::HygMX pSP-AC

This study

Diploid strain transformed with plasmids PSP-AC-GSH1

H1-GSH1

H2-GSH1

H3-GSH1

H4-GSH1

2421_C1-GSH1

E5_C2-GSH1

*During research we noticed that starting YIIc17_E5 strains were also histidine auxotrophs that could not be complemented with a functional HIS3 gene

${ }^{*} \mathrm{H} 1$ and $\mathrm{H} 4$ were isolated as separate random colonies that grew on selective media after mating of 2421REDa and with YIlc17_E5a

${ }^{* * *} \mathrm{H} 2$ and $\mathrm{H} 3$ were isolated as separate random colonies that grew on selective media after mating of E5REDa and with UWOPS87-2421a 


\section{Plasmid retention under non-selective conditions}

To determine the retention of plasmids in non-selective conditions, strains transformed with replicative plasmid pSP-YAP1 containing URA3 gene as a selectable marker (Table S1) were first grown in selective SC-Ura liquid medium until the stationary phase, and then they were diluted and inoculated in four test tubes containing rich YPD medium. One test tube was used as a control, while in the other test tubes growth inhibitors were added to the final concentration of $100 \mathrm{mM}$ (acetic and levulinic acids) or to final concentration of $20 \mathrm{mM}$ (2-furaldehyde). After two days in the shaker at $28{ }^{\circ} \mathrm{C}$, cultures were diluted and fifth and sixth decimal dilutions were plated on YPD medium. Colonies that grew on YPD medium were replica plated on SC-Ura medium and the percentage of Ura ${ }^{+}$cells that retained replicative plasmid was calculated.

\section{RESULTS AND DISCUSSION}

Lignocellulosic waste is abundant but, due to its complex chemical composition, yeasts cannot use it directly for fermentation and bioethanol production. Therefore, lignocellulosic materials need to be pretreated to release fermentable sugars, but during pretreatment aliphatic acids and furan derivates, which inhibit growth and fermentation, are also formed. Therefore, apart from high ethanol concentration, bioethanol producer needs to be resistant to growth and fermentation inhibitors.

The aim of this study was to investigate whether the construction of Saccharomyces cerevisiae intraspecies hybrids by mating parental haploids having desirable traits is a promising approach for construction of bioethanol producer. To achieve this, we used two natural strains of S. cerevisiae, UWOPS87-2421 that tolerates acetic and levulinic acids and was found in the flower of the cactus Opuntia megacantha in Hawaii, and Yllc17_E5, isolated from wine in France that has been shown to be sensitive to formic acid, 2-furaldehyde and 5-hydroxymethylfurfural (33,34).

To expedite the construction of hybrid $(\mathrm{H} 1-\mathrm{H} 4)$ and control (2421_C1 and E5_C2) diploids by mating of haploids, UWOPS87-2421a and Yllc17_E5a strains were first modified by plasmid integration. Afterwards, constructed diploids were analysed for their ability to produce $\mathrm{CO}_{2}$ by fast fermentation test on BTB plates and to grow in the presence of growth and fermentation inhibitors. Additionally, the strains were transformed with replicative plasmids carrying yeast ATR1, FLR1 and YAP1 genes previously shown to increase the resistance of strains to growth and fermentation inhibitors.
Low gene targeting fidelity in UWOPS87-2421a and YIIc17_E5a S. cerevisiae strains

Starting a- and a-mating parental haploids had the same $\mathrm{Ade}^{+}$Ura phenotype (Fig. 1 and Table $1(24,25)$ ). To facilitate the construction of diploid strains by mating, a-mating strains were first modified by targeted integration of the plasmid pRED150 in ADE2 gene, changing their phenotype to Ade- Ura ${ }^{+}$. Furthermore, such transformants can be easily recognised because inactivation of ADE2 gene produces red colonies (35).

Surprisingly, during construction of 2421REDa and E5REDa strains, phenotypic analyses of transformants revealed significantly lower percentage of transformants carrying targeted plasmid integration than in standard S. cerevisiae laboratory strains. Thus, the molecular analysis by Southern blotting confirmed that success of targeted plasmid integration (gene targeting fidelity) is only 25 and $1.7 \%$ in UWOPS-2421a and YIIC_E5a strains, respectively ( $p<0.0001$, Fig. 1 and Table 2), whereas in standard S. cerevisiae it is as high as $98 \%$ (28). Apart from low gene targeting fidelity in UWOPS-2421a and YIIC_E5a strains, the percentage of multiple targeted plasmid integration also seems to be high (around $50 \%$ in comparison to $10 \%$ in standard S. cerevisiae laboratory strains during spheroplast transformation (36)). In addition, molecular analysis of white $\mathrm{Ade}^{+} \mathrm{Ura}^{+}$transformants revealed spectra of aberrant (off-targeted) genetic events (Table 2). We have previously shown that aberrant (off-targeting) transformation events in plasmid integration assays are: (i) integration of plasmid molecule in the random position of the host genome (illegitimate integration), and (ii) targeted chromosome duplication (TCD) resulting in heteroallelic transformants - aneuploids having at least two copies of targeted chromosome, one carrying untransformed allele and the other carrying the allele expected after successful gene targeting (28).

Concerning spectra of off-targeted events in UWOPS-2421a and YIIC_E5a strains (3.6-16.7\% of illegitimate integration and 83.3-96.4\% of TCD), they are similar to the spectra in standard S. cerevisiae laboratory strains. However, due to low gene targeting fidelity, and considering all analysed transformants, TCD is the most frequent genetic event, being higher than 60 and $90 \%$ for UWOPS-2421a and YIIC_E5a strains, respectively. This could be a consequence of chromosome duplication during gene targeting (TCD) but also the existence of at least two copies of chromosome XV carrying ADE2 gene in UWOPS-2421a and YIIC_E5a strains prior to transformation. However, a qPCR analysis of chromosome copy number

Table 2. Spectra of genetic events in strains UWOPS87-2421a and YIIC17_E5a during gene targeting with pRED150 plasmid

\begin{tabular}{|c|c|c|c|c|c|}
\hline Genetic event & UWOPS87-2421a & Yllc17_E5a & Genetic event & UWOPS87-2421a & Yllc17_E5a \\
\hline \multirow{2}{*}{$\begin{array}{l}\text { Successful gene targeting } \\
\text { (percentage of red, Ade } \\
\text { Ura }{ }^{+} \text {transformants) }\end{array}$} & \multirow{2}{*}{$22 / 88(25.0 \%)$} & \multirow{2}{*}{ 2/115 (1.7\%) } & Single plasmid integration & $12 / 22(54.5 \%)$ & $1 / 2(50.0 \%)$ \\
\hline & & & Multiple plasmid integration & $10 / 22(45.4 \%)$ & $1 / 2(50.0 \%)$ \\
\hline \multirow{2}{*}{$\begin{array}{l}\text { Off-targeted events } \\
\text { (percentage of white, Ade } \\
\text { Ura }{ }^{+} \text {transformants) }\end{array}$} & \multirow{2}{*}{ 66/88 (75.0 \%) } & \multirow{2}{*}{ 113/115 (98.3 \%) } & $\begin{array}{l}\text { Illegitimate plasmid } \\
\text { integration }\end{array}$ & 1/6 (16.7 \%) & $1 / 28(3.6 \%)$ \\
\hline & & & $\begin{array}{c}\text { Targeted chromosome } \\
\text { duplication }\end{array}$ & $5 / 6(83.3 \%)$ & $27 / 28(96.4 \%)$ \\
\hline Analysed transformants & 88 & 115 & Analysed transformants & 28 & 30 \\
\hline
\end{tabular}


in these two strains revealed haploid DNA content (data not shown). Therefore, frequent TCD in these strains could be a consequence of some mutations, change in gene expression and/or protein activity that remains to be identified. From this point of view, it should be noted that fidelity of gene targeting and spectrum of genetic events strongly depend on the transformation procedure used to deliver the transforming DNA to the yeast cell (30) as well as on the influence of e.g. EXO1 and SGS1 genes on both transformation efficiency and gene targeting fidelity $(28,37)$.

\section{Isogenic hybrids display phenotypic diversity and different fermentation activity}

First, by crossing a-mating Ade- Ura $^{+}$cells with a-mating $\mathrm{Ade}^{+}$Ura cells, we constructed hybrid and control diploids with $\mathrm{Ade}^{+}$Ura ${ }^{+}$phenotype. In the second step, a loss of pRED150 plasmid from the ade2 region in the genome resulted in restoring parental $\mathrm{Ade}^{+}$Ura- genotype, enabling comparison of parental haploids and constructed diploids (Fig. $2(20,21)$ ).

Constructed diploids (H1-H4, 2421_C1 and E5_C2) and parental haploids (UWOPS87-2421a and YIIc17_E5a) were subjected to semi-quantitative test for ethanol production on bromothymol blue (BTB) plates. As described previously (31,32), the plates containing an indicator BTB can be used for semi-quantitative monitoring of fermentation activity of a strain because the production of $\mathrm{CO}_{2}$, due to fermentation, decreases $\mathrm{pH}$. As the $\mathrm{pH}$ decreases, the colour of BTB and media containing it changes from green to yellow or even deep orange/red. Moreover, deep orange or red colour of the BTB medium and even colonies was observed with yeast strains that are frequently used for the production of wines, indicating their better fermentation ability (31,32). As a continuation of this research, batch ethanol production in fermentation flasks or laboratory scale bioreactors will be performed to quantify ethanol titres and yields obtained by newly constructed intraspecies hybrid strains.

The test of fermentative potential showed that hybrids $\mathrm{H} 1$ $-\mathrm{H} 4$, although isogenic and originating from the same parental strains, have different fermentative ability, whereas, as expected, control haploid Yllc17_E5a and diploid E5_C2 strains (isolated from wine) have the highest fermentation activity (Fig. 3). Additionally, when different hybrid cultures were diluted and plated on BTB plates to allow for the growth of single colonies, it was observed that hybrids $\mathrm{H} 1$ and $\mathrm{H} 4$ formed colonies of uniform colour and size, while hybrids $\mathrm{H} 2$ and $\mathrm{H} 3$ formed colonies of different size and colour indicating phenotypic variability among descendants of a single heterozygous diploid cell (Fig. 3). This suggested that hybrids that originated from a cross of E5REDa and UWOPS87-2421a experience genome instability and it is in agreement with the already observed genetic instability of intraspecies hybrids used for wine production, which could explain differences in both the amount of produced ethanol and strain stability (38-40). More importantly, phenotypic diversity of constructed intraspecies hybrids could allow further selection of properties useful for bioethanol production on different lignocellulosic hydrolysates. a)

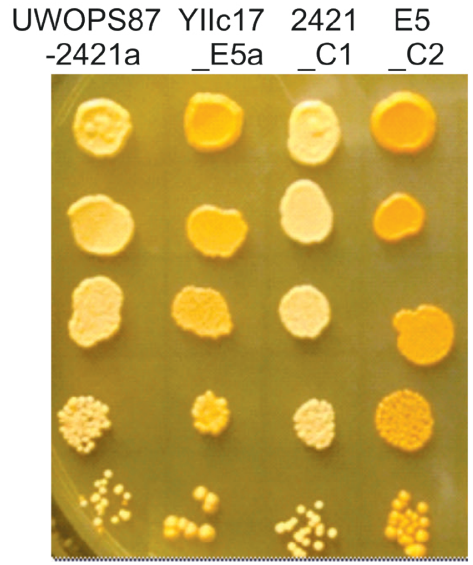

b)

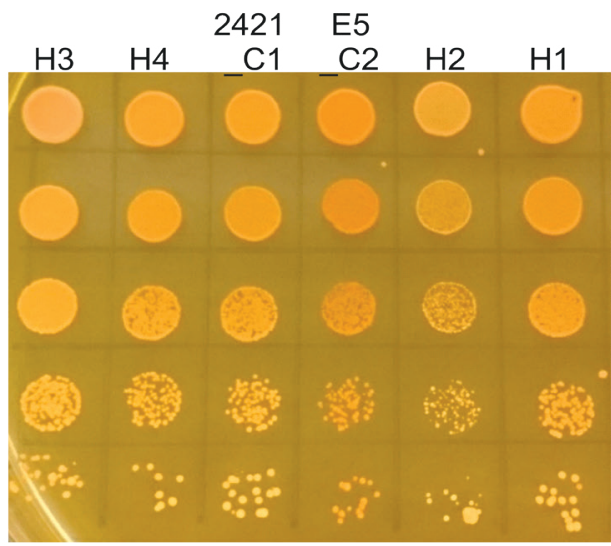

c)

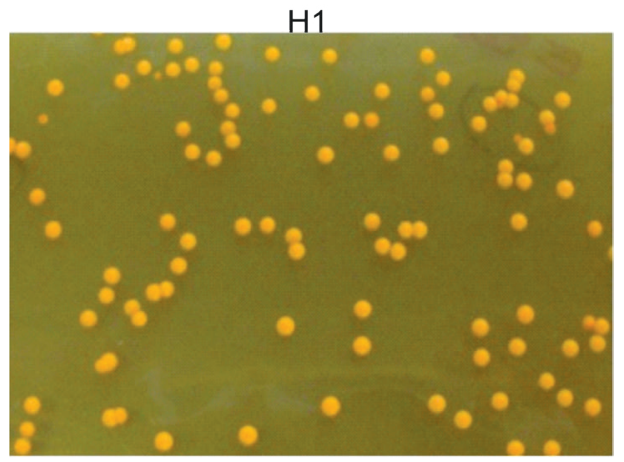

d)

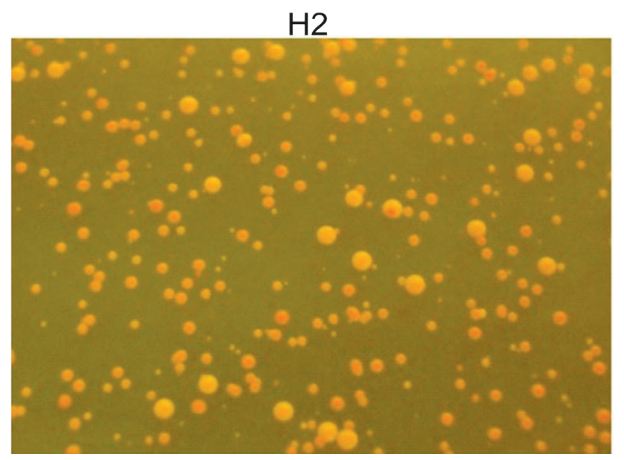

Fig. 3. Fermentative potential of yeast strains assessed by semi-quantitative method using bromothymol blue (BTB) plates. Comparison of: a) parental haploids and parental diploids, b) hybrids $(\mathrm{H} 1-\mathrm{H} 4)$ and control diploid strains (2421_C1 and E5_C2), colonies obtained from the single colony of c) H1 (2421REDa $\times$ YIIC17_E5a) and d) H2 (E5REDa $\times$ UWOPS87-2421a) diploids, respectively 


\section{Growth and survival of strains in the presence of growth and fermentation inhibitors}

Growth of the constructed heterozygous hybrids $\mathrm{H} 1$ -H4 and control diploids 2421_C1 and E5_C2 and their performance in media containing inhibitors frequently found in lignocellulosic hydrolysates were analysed in liquid and on solid media. First, single growth and fermentation inhibitors (acetic and levulinic acids, 2-furaldehyde and ethanol) were added to liquid complex complete YPD medium (Fig. 4). The obtained results clearly show that hybrids $\mathrm{H} 1$ and $\mathrm{H} 2$ grew faster both in the presence and in the absence of acetic and levulinic acids than controls (2421_C1 and E5_C2, Fig. 4). At lower concentrations of 2-furaldehyde (10 mM), hybrid $\mathrm{H} 1$ grew the best, while further increase of the concentration equally inhibited the growth of all strains (Fig. 4). Ethanol, in volume fractions used here, severely affected all tested strains, although at $10 \%$ the best growth was observed of control 2421_C1 and hybrid H2 strains (Fig. 4). The growth of the other two hybrids ( $\mathrm{H} 3$ and $\mathrm{H} 4)$ in the presence of growth and fermentation inhibitors was also tested, but these strains did not perform as well as $\mathrm{H} 1$ and $\mathrm{H} 2$ and were excluded from further studies.

Additionally, it was noticed that control 2421_C1 and hybrid $\mathrm{H} 2$ always form big floccules, aggregates that have been shown to protect cells from harsh environment (41). Although flocculation is still not completely understood, it is influenced by temperature, $\mathrm{pH}(42,43)$, osmotic stress, ethanol concentration, nutrient availability and the presence of calcium and zinc ions (41). While the flocculation of laboratory strains is unwanted, it positively influences production of bioethanol, heterologous proteins and bioremediation of heavy metals. Moreover, in the industrial setting, flocculation usually starts at a specific phase of culture growth or fermentation and allows easier separation of the producer strain at the end of the process.

Survival in the presence of inhibitors was also tested on YPD, SC or SC-Ura media that contained acetic and levulinic acids and 2-furaldehyde (Fig. 5). Hybrid $\mathrm{H} 1$ had the highest survival on rich YPD plates containing $80 \mathrm{mM}$ acetic acid and $5 \mathrm{mM}$ 2-furaldehyde, while on the plates containing $140 \mathrm{mM}$ levulinic acid the survival of hybrids $\mathrm{H} 1$ and $\mathrm{H} 2$ was almost the same (Fig. 5). As expected, the change of medium composition significantly influenced the growth and survival of all strains. Thus, the survival of all strains on SC plates containing $80 \mathrm{mM}$ acetic and levulinic acids significantly decreased, while the change of medium had only a minor effect on the survival of strains on the SC plates containing $5 \mathrm{mM} 2$-furaldehyde, and hybrid $\mathrm{H} 1$ again had the highest survival (Fig. 5).

Although concentrations of acetic and levulinic acids and 2 -furaldehyde in lignocellulosic hydrolysates vary $(4,5,7)$, they are lower than those used here. The presented results show that levulinic acid has lower inhibitory effect on the growth of yeast strains than acetic acid (Fig. 4 and Fig. 5). This is in contrast to higher toxic effect of levulinic acid reported by Jönsson et al. (4), explained by easier entrance to cells (44). Still, Mirisola and Longo (45) suggested that the $\mathrm{pH}$ and the exact composition of the medium have a significant impact on yeast growth and ageing, and that intracellular drop of $\mathrm{pH}$ results in a)

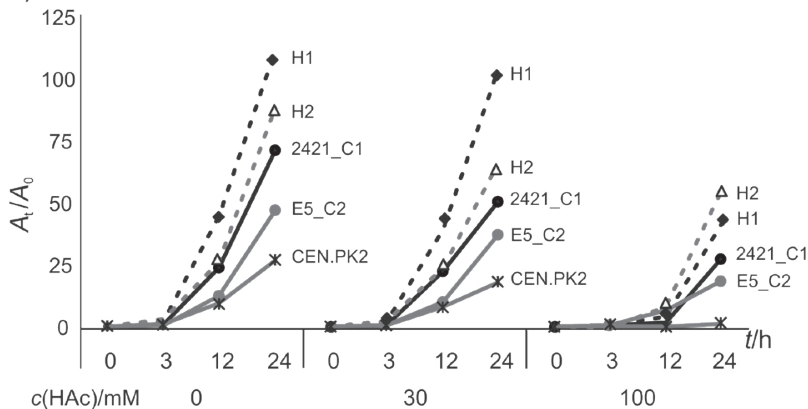

b)

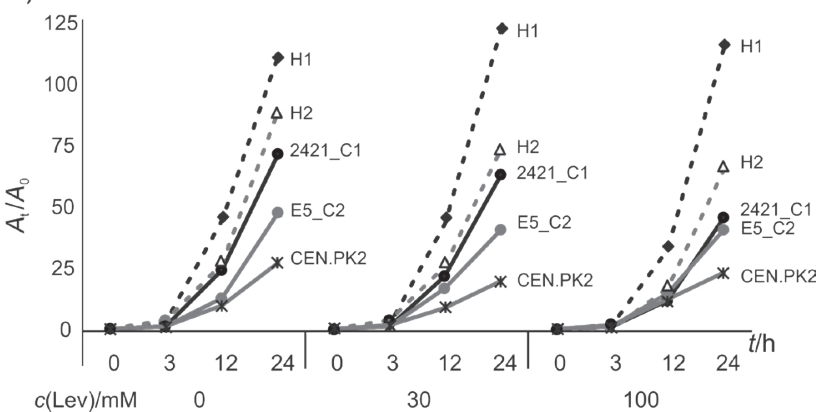

c)

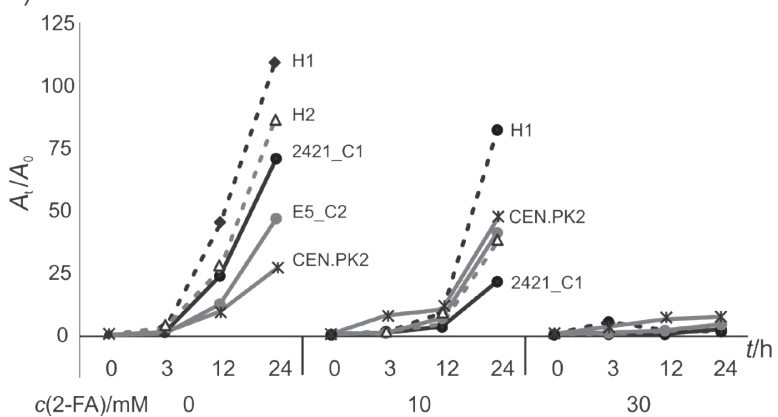

d)

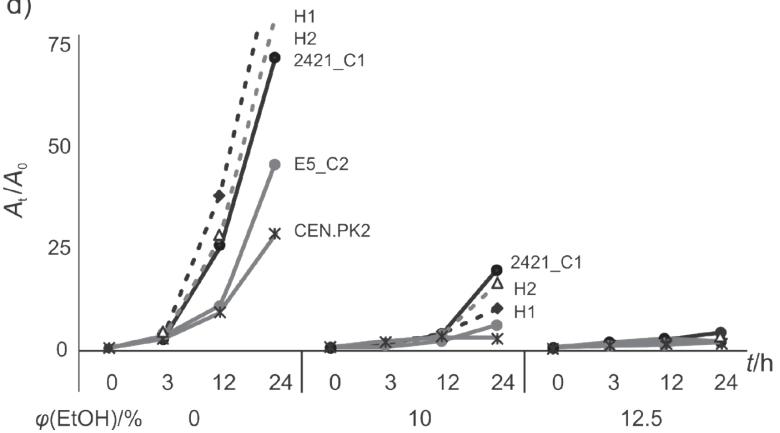

Fig. 4. Growth of heterozygous hybrid ( $\mathrm{H} 1$ and $\mathrm{H} 2)$ and homozygous control (2421_C1 and E5_C2) strains in liquid YPD medium containing: a) acetic acid, b) levulinic acid, c) 2-furaldehyde and d) ethanol. The results are expressed as the ratio of the absorbance $\left(A_{t}\right)$ at a particular time $(t=3$, 12 and $24 \mathrm{~h}$ ) and the absorbance $\left(A_{0}\right)$ at the beginning of the experiment ( $\left.t=0 \mathrm{~h}\right)$. CEN.PK2 strain was used as an additional control 

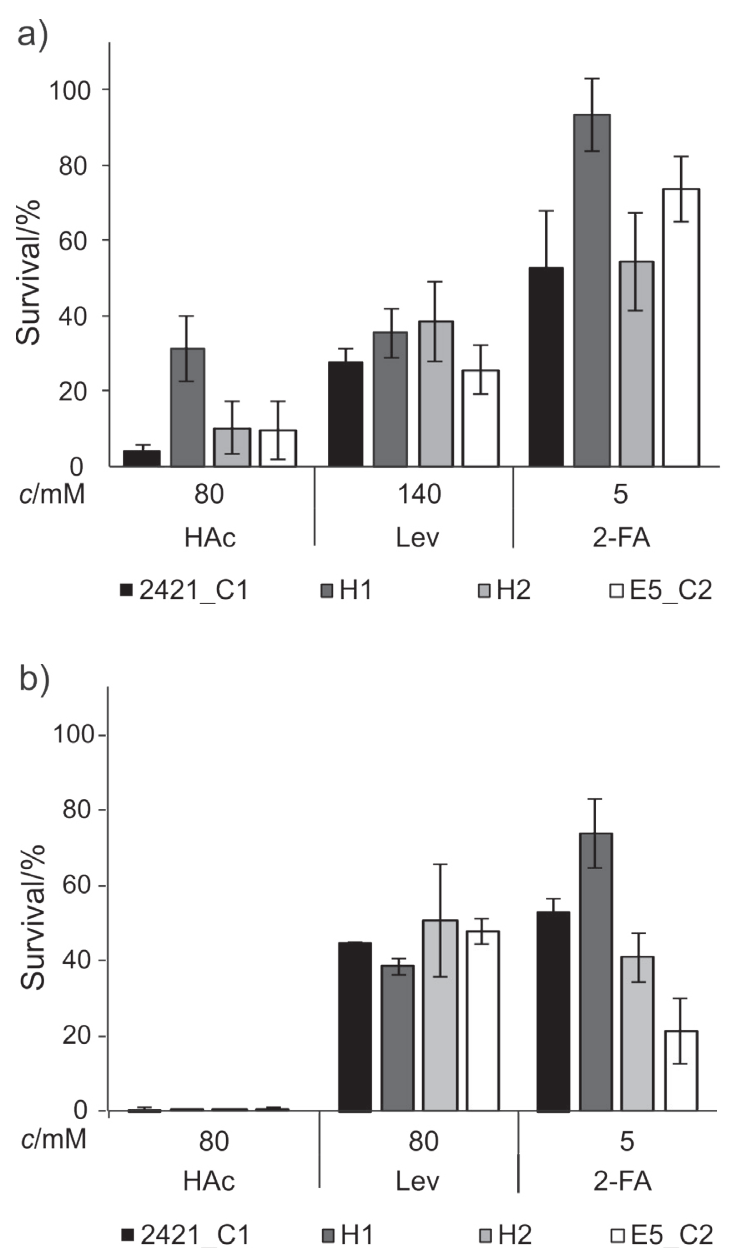

Fig. 5. Survival of heterozygous hybrid $(\mathrm{H} 1$ and $\mathrm{H} 2)$ and homozygous control (2421 C1 and E5 C2) strains on: a) complex complete (YPD) and b) synthetic complete (SC) solid media containing acetic (HAC) and levulinic (Lev) acids and 2-furaldehyde (2-FA). Results are expressed according to the $100 \%$ strain survival on the corresponding medium (YPD or SC) without inhibitors. Error bars represent standard deviation

the production of reactive oxygen species. In the scope of this work, it is important to mention that Burtner et al. (46) suggested that acetic acid is one of the most important factors that induce yeast ageing and cell death, while Heer and Sauer (7) identified 2-furaldehyde as a main toxic compound that inhibits both cell growth and ethanol production, and this has been confirmed by the results presented here (Fig. 4 and Fig. 5). Although yeasts can tolerate up to $18 \%$ of ethanol, ethanol volume fractions higher than $10 \%$, especially in the presence of 2-furaldehyde, have a significant inhibitory effect (4).

To investigate the influence of overexpression of yeast ATR1, YAP1, FLR1 and GSH1 genes on the survival in the presence of inhibitors, all 36 strains transformed with replicative plasmids carrying URA3 gene as selectable marker and either ATR1, YAP1, FLR1 or GSH1 gene under the regulation of TEF1 or PGK1 promoter (Table S1) were tested on SC-Ura plates (to prevent the loss of replicative plasmids) containing $60 \mathrm{mM}$ acetic acid, 100 mM levulinic acid or 5 mM 2-furaldehyde (Fig. 6).
To assure the overexpression, ATR1, YAP1 and FLR1 genes were cloned in PSP-G2 plasmid which is present in approx. 50 copies per cell because it contains $2 \mu$ origin of replication $(47,48)$, whereas GSH1 gene was cloned in centromeric PSP-AC plasmid since the positive effect of the GSH1 gene was observed only if it was present in several copies per cell (17). However, in this work overexpression of FLR1 and GSH1 genes did not have any significant influence (data not shown), while overexpression of YAP1 gene had overall more positive effect on the survival of the strains in the presence of growth inhibitors than the overexpression of ATR1 gene (Fig. 6). Beneficial effect of overexpression of YAP1 gene can be explained by the fact that it encodes a transcription factor for various genes involved in the response to oxidative and heavy metal stress (49-52). Positive but less pronounced effect of the
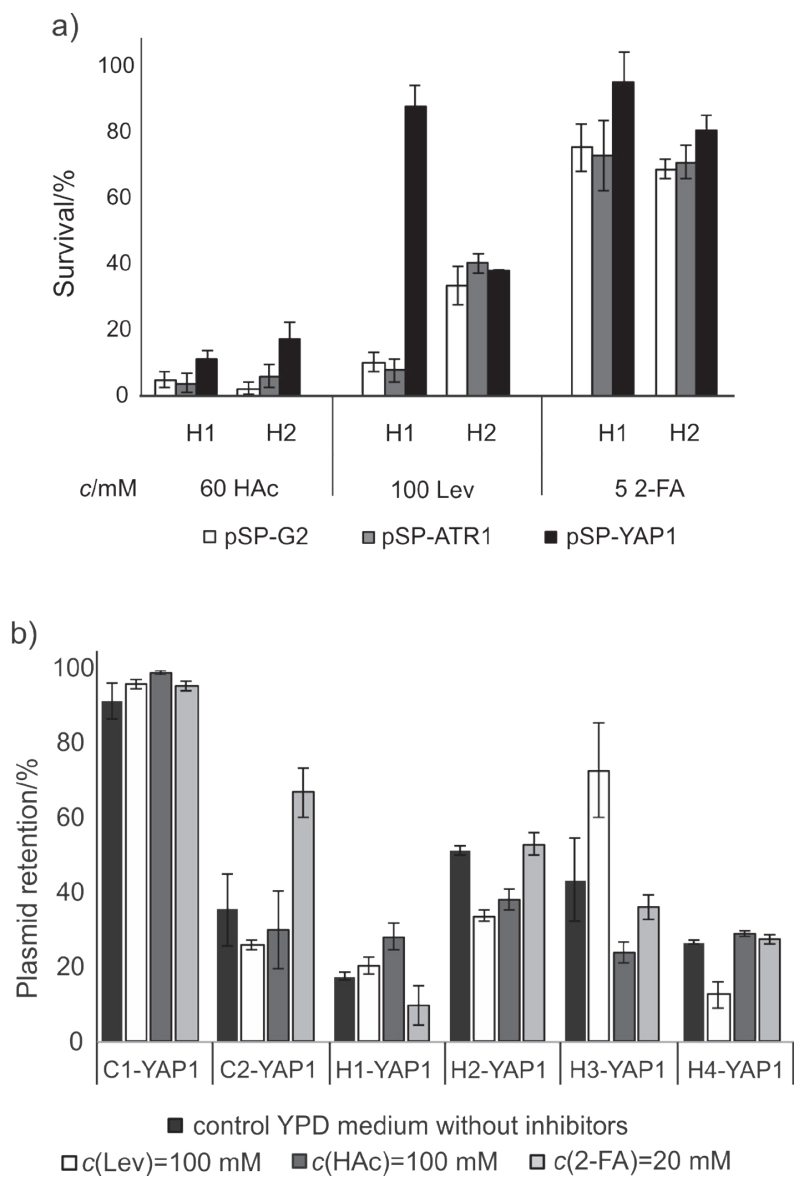

Fig 6. Survival of the hybrid strains overexpressing YAP1 and ATR1 genes and retention of the plasmid PSP-YAP1 in diploid strains in nonselective conditions: a) survival of the hybrid strains $\mathrm{H} 1$ and $\mathrm{H} 2$ containing vector PSP-G2 or plasmids PSP-ATR1 and pSP-YAP1 overexpressing ATR1 and YAP1 genes on solid SC-Ura medium in the presence of acetic (HAC) and levulinic (Lev) acids and 2-furaldehyde (2-FA). Results are expressed according to the $100 \%$ strain survival on the SC-Ura medium without inhibitors; error bars represent standard deviation; and $b$ ) retention of the plasmid pSP-YAP1 overexpressing YAP1 gene in heterozygous hybrid $(\mathrm{H} 1-\mathrm{H} 4)$ and homozygous control (2421_C1 and E5_C2) diploid strains grown in YPD medium containing acetic and levulinic acids and 2-furaldehyde. The percentage of cells which retained the plasmid after $48 \mathrm{~h}$ of cultivation is shown; error bars represent standard deviation 
overexpression of ATR1 gene (Fig. 6) could be a consequence of a direct involvement of Atr1 protein in the formation of transmembrane pumps (53).

Since the YAP1 gene had the most positive influence on the survival of yeast strains in the presence of inhibitors in SC-Ura medium (see above), it was investigated if the growth inhibitor can be used as the only selective pressure for the retention of the replicative plasmid carrying YAP1 gene (Fig. 6). The results showed that the presence of the inhibitors did not result in higher frequency of plasmid retention. However, it is interesting that retention of the PSP-YAP1 plasmid in the 2421_C1 strain was as high as $90 \%$ both in the presence and absence of inhibitors.

The newly constructed intraspecies hybrids described here survive better than the parental strains in complex and synthetic media containing different single inhibitors of growth and fermentation. Since lignocellulosic hydrolysates used for bioethanol production contain several growth and fermentation inhibitors that frequently have synergistic effect on a producer yeast strain, we plan to challenge hybrid strains by inoculating them both in complex and synthetic media containing acetic and levulinic acids and 2-furaldehyde, and in lignocellulosic hydrolysates.

\section{CONCLUSIONS}

Construction of intraspecies hybrids by mating of natural isolates having desirable traits is a promising approach in the development of biotechnologically applicable Saccharomyces cerevisiae strains. In this work such approach was used during development of bioethanol producer and it was found that the constructed heterozygous diploids display genetic instability, resulting in different fermentation ability, allowing further improvement by selection of desirable biotechnological characteristics. Some of the constructed hybrids showed better performance in the presence of several growth and fermentation inhibitors, frequently present in lignocellulosic hydrolysates, which was further increased by the overexpression of the YAP1 gene.

\section{ACKNOWLEDGEMENTS}

We would like to thank the collaborators on the project "Sustainable production of bioethanol and biochemicals from agricultural waste lignocellulosic raw materials" on insightful discussions.

\section{FUNDING}

This work was supported by Croatian Science Foundation grant IP-11-2013-9158-SPECH-LRM (Sustainable production of bioethanol and biochemicals from agricultural waste lignocellulosic raw materials) to Božidar Šantek.

\section{SUPPLEMENTARY MATERIAL}

All supplementary material is available at www.ftb.com.hr.

\section{REFERENCES}

1. Nigam PS, Singh A. Production of liquid biofuels from renewable resources. Prog Energy Combust Sci. 2011;37(1):52-68. https://doi.org/10.1016/j.pecs.2010.01.003

2. Jäger $G$, Büchs J. Biocatalytic conversion of lignocellulose to platform chemicals. Biotechnol J. 2012;7(9):1122-36.

https://doi.org/10.1002/biot.201200033

3. Palmqvist E, Hahn-Hägerdal B. Fermentation of lignocelullosic hydrolysates. II: Inhibitors and mechanisms of inhibition. Bioresour Technol. 2000;74(1):25-33.

https://doi.org/10.1016/S0960-8524(99)00161-3

4. Jönsson LJ, Alriksson B, Nilvebrant NO. Bioconversion of lignocelullose: Inhibitors and detoxification. Biotechnol Biofuels. 2013;6:16.

https://doi.org/10.1186/1754-6834-6-16

5. Adeboye PT, Bettiga M, Olsson L. The chemical nature of phenolic compounds determines their toxicity and induces distinct physiological responses in Saccharomyces cerevisiae in lignocellulose hydrolysates. AMB Express. 2014;4:46. https://doi.org/10.1186/s13568-014-0046-7

6. Palmqvist E, Almeida JS, Hahn-Hägerdal B. Influence of furfural on anaerobic glycolytic kinetics of Saccharomyces cerevisiae in batch culture. Biotechnol Bioeng. 1999;62(4):447-54. https://doi.org/10.1002/(SICI)10970290(19990220)62:4<447::AID-BIT7>3.0.CO;2-0

7. Heer $D$, Sauer U. Identification of furfural as a key toxin in lignocellulosic hydrolysates and evolution of a tolerant yeast strain. Microb Biotechnol 2008;1(6):497-506. https://doi.org/10.1111/j.1751-7915.2008.00050.x

8. Alexandre H, Ansanay-Galeote V, Dequin S, Blondin B. Global gene expression during short-term ethanol stress in Saccharomyces cerevisiae. FEBS Lett. 2001;498(1):98-103.

https://doi.org/10.1016/S0014-5793(01)02503-0

9. Klinke HB, Thomsen $A B$, Ahring BK. Inhibition of ethanol-producing yeast and bacteria by degradation products produced during pre-treatment of biomass. Appl Microbiol Biotechnol. 2004;66(1):10-26.

https://doi.org/10.1007/s00253-004-1642-2

10. Palmqvist E, Grage H, Meinander NQ, Hahn-Hägerdal B. Main and interaction effects of acetic acid, furfural, and p-hydroxybenzoic acid on growth and ethanol productivity of yeasts. Biotechnol Bioeng. 1999;63(1):46-55.

https://doi.org/10.1002/(SICI)10970290(19990405)63:1<46::AID-BIT5>3.0.CO;2-J

11. Pereira FB, Romaní A, Ruiz HA, Teixeira JA, Domingues L. Industrial robust yeast isolates with great potential for fermentation of lignocellulosic biomass. Bioresour Technol. 2014;161:192-9.

https://doi.org/10.1016/j.biortech.2014.03.043

12. Narayanan V, Sànchez i Nogué V, van Niel EWJ, Gorwa-Grauslund MF. Adaptation to low $\mathrm{pH}$ and lignocellulosic inhibitors resulting in ethanolic fermentation and growth 
of Saccharomyces cerevisiae. AMB Express. 2016;6:59.

https://doi.org/10.1186/s13568-016-0234-8

13. Narayanan V, Schelin J, Gorwa-Grauslund M, van Niel EWJ, Carlquist M. Increased lignocellulosic inhibitor tolerance of Saccharomyces cerevisiae cell populations in early stationary phase. Biotechnol Biofuels. 2017;10:114.

https://doi.org/10.1186/s13068-017-0794-0

14. Larsson S, Cassland P, Jönsson LJ. Development of a Saccharomyces cerevisiae strain with enhanced resistance to phenolic fermentation inhibitors in lignocelullose hydrolisates by heterologous expression of laccase. Appl Environ Microbiol. 2001;67(3):1163-70.

https://doi.org/10.1128/AEM.67.3.1163-1170.2001

15. Hasunuma T, Sanda T, Yamada R, Yoshimura K, Ishii J, Kondo A. Metabolic pathway engineering based on metabolomics confers acetic and formic acid tolerance to a recombinant xylose-fermenting strain of Saccharomyces cerevisiae. Microb Cell Fact. 2011;10:2. https://doi.org/10.1186/1475-2859-10-2

16. Alriksson B, Sárvári Horváth I, Jönsson LJ. Overexpression of Saccharomyces cerevisiae transcription factor and multidrug resistance genes conveys enhanced resistance to lignocellulose-derived fermentation inhibitors. Process Biochem. 2010;45(2):264-71.

https://doi.org/10.1016/j.procbio.2009.09.016

17. Ask M, Mapelli V, Höck H, Olsson L, Bettiga M. Engineering glutathione biosynthesis of Saccharomyces cerevisiae increases robustness to inhibitors in pretreated lignocelullosic materials. Microb Cell Fact. 2013;12:87.

https://doi.org/10.1186/1475-2859-12-87

18. Miklenić M. Recombinogenicity of palindromes and quasipalindromes in the yeast Saccharomyces cerevisiae genome [PhD Thesis]. Zagreb, Croatia: University of Zagreb; 2015 (in Croatian).

19. Lisnić B, Svetec IK, Štafa A, Zgaga Z. Size-dependent palindrome-induced intrachromosomal recombination in yeast. DNA Repair (Amst). 2009;8(3):383-9. https://doi.org/10.1016/j.dnarep.2008.11.017

20. Boeke JD, La Croute F, Fink GR. A positive selection for mutants lacking orotidine-5'-phosphate decarboxylase activity in yeast: 5-fluoroorotic acid resistance. Mol Gen Genet. 1984;197(2):345-6.

https://doi.org/10.1007/BF00330984

21. Svetec IK, Stjepandić D, Borić V, Mitrikeski PT, Zgaga Z. The influence of a palindromic insertion on plasmid integration in yeast. Food Technol Biotechnol 2001;39(3):169-73.

22. Partow S, Siewers V, Bjørn S, Nielsen J, Maury J. Characterization of different promoters for designing a new expression vector in Saccharomyces cerevisiae. Yeast. 2010;27(11):955-64.

https://doi.org/10.1002/yea.1806
23. Sambrook J, Russell DW, editors. Molecular cloning: A laboratory manual. Cold Spring Harbor, NY, USA: Cold Spring Harbor Laboratory Press; 2001.

24. Cubillos FA, Louis EJ, Liti G. Generation of a large set of genetically tractable haploid and diploid Saccharomyces strains. FEMS Yeast Res. 2009;9(8):1217-25. https://doi.org/10.1111/j.1567-1364.2009.00583.x

25. Entian KD, Kötter P. 25 Yeast genetic strain and plasmid collections. In: Stansfield I, Stark MJR, editors. Methods in Microbiology, vol. 36. NY, USA: Elsevier Ltd.; 2007. pp. 629-66. https://doi.org/10.1016/S0580-9517(06)36025-4

26. Sherman F. Getting started with yeast. Methods Enzymol. 2002;350:3-41.

https://doi.org/10.1016/S0076-6879(02)50954-X

27. Pavelka N, Rancati G, Zhu J, Bradford WD, Saraf A, Florens $\mathrm{L}$, et al. Aneuploidy confers quantitative proteome changes and phenotypic variation in budding yeast. Nature. 2010;468(7321):321-5. https://doi.org/10.1038/nature09529

28. Štafa A, Miklenić M, Žunar B, Lisnić B, Symington LS, Svetec IK. Sgs1 and Exo1 suppress targeted chromosome duplication during ends-in and ends-out gene targeting. DNA Repair (Amst). 2014;22:12-23.

https://doi.org/10.1016/j.dnarep.2014.07.004

29. Gjuračić K, Zgaga Z. Illegitimate integration of single-stranded DNA in Saccharomyces cerevisiae. Mol Gen Genet. 1996;253(1-2):173-81.

https://doi.org/10.1007/s004380050310

30. Štafa A, Svetec Miklenić M, Zandona A, Žunar B, Čadež N, Petković $\mathrm{H}$, Svetec IK. In Saccharomyces cerevisiae gene targeting fidelity depends on a transformation method and proportion of the overall length of the transforming and targeted DNA. FEMS Yeast Res. 2017;17(4):fox041.

https://doi.org/10.1093/femsyr/fox041

31. Robillard R. A microbial breathalyzer: Design of a colorimetric assay for the detection and quantification of ethanol production in microbes [MSc Thesis]. Worchester, MA, USA: Worchester Polytechnic Institute; 2007.

32. Peet KC. Optimizing microbial ethanol: Carbon source influence and detrimental genes for ethanol production [MSC Thesis]. Worchester, MA, USA:Worchester Polytechnic Institute; 2008.

33. Liti G, Carter DM, Moses AM, Warringer J, Parts L, James SA, et al. Population genomics of domestic and wild yeasts. Nature. 2009;458(7236):337-41.

https://doi.org/10.1038/nature07743

34. Wimalasena TT, Greetham D, Marvin ME, Liti G, Chandelia Y, Hart A, et al. Phenotypic characterisation of Saccharomyces spp. yeast for tolerance to stresses encountered during fermentation of lignocellulosic residues to produce bioethanol. Microb Cell Fact. 2014;13:47.

https://doi.org/10.1186/1475-2859-13-47 
35. Jones EW, Fink GR. Regulation of amino acid and nucleotide biosynthesis in yeast. In: Strathern JN, Jones EW, Broach JR, editors. The molecular biology of the yeast Saccharomyces: Metabolism and gene expression. Cold Spring Harbor, NY, USA: Cold Spring Harbor Laboratory Press; 1982. pp. 181-299.

36. Svetec IK, Štafa A, Zgaga Z. Genetic side effects accompanying gene targeting in yeast:The influence of short heterologous termini. Yeast. 2007;24(8):637-52. https://doi.org/10.1002/yea.1497

37. Štafa A, Svetec IK, Zgaga Z. Inactivation of the SGS1 and EXO1 genes synergistically stimulates plasmid Integration in yeast. Food Technol Biotechnol. 2005;43(2):103-8.

38. Kunicka-Styczyńska A, Rajkowska K. Physiological and genetic stability of hybrids of industrial wine yeasts Saccharomyces sensu stricto complex. J Appl Microbiol. 2011;110(6):1538-49.

https://doi.org/10.1111/j.1365-2672.2011.05009.x

39. Kunicka-Styczyńska A, Rajkowska K. Phenotypic and genotypic diversity of wine yeasts used for acidic musts. World J Microbiol Biotechnol. 2012;28(5):1929-40. https://doi.org/10.1007/s11274-011-0994-X

40. Kumaran R, Yang SY, Leu JY. Characterization of chromosome stability in diploid, polyploid and hybrid yeast cells. PLoS ONE. 2013;8(7):e68094.

https://doi.org/10.1371/journal.pone.0068094

41. Soares EV. Flocculation in Saccharomyces cerevisiae: A review. J Appl Microbiol. 2010;110(1):1-18.

https://doi.org/10.1111/j.1365-2672.2010.04897.x

42. Verstrepen KJ, Derdelinckx G, Verachtert H, Delvaux FR. Yeast flocculation: What brewers should know. Appl Microbiol Biotechnol. 2003;61(3):197-205. https://doi.org/10.1007/s00253-002-1200-8

43. Verstrepen KJ, Klis FM. Floculation, adhesion and biofilm formation in yeasts. Mol Microbiol. 2006;60(1):5-15. https://doi.org/10.1111/j.1365-2958.2006.05072.x

44. Soccol CR, Faraco V, Karp S, Vandenberghe LPS, Thomaz-Soccol V, Woiciechowski A, Pandey A. Lignocelullosic bioethanol: Current status and future perspectives. In: Pandey A, Larroche C, Ricke SC, Dussap CG, Gnansounou E, editors. Biofuels. Amsterdam, The Netherlands: Academic Press; 2011. pp. 101-22.

https://doi.org/10.1016/B978-0-12-385099-7.00005-X

45. Mirisola MG, Longo VD. Acetic acid and acidification accelerate chronological and replicative aging in yeast. Cell Cycle. 2012;11(19):3532-3.

https://doi.org/10.4161/cc.22042

46. Burtner CR, Murakami CJ, Kennedy BK, Kaeberlein M. A molecular mechanism of chronological aging in yeast. Cell Cycle. 2009;8(8):1256-70.

https://doi.org/10.4161/cc.8.8.8287

47. Ludwig DL, Bruschi CV. The 2-micron plasmid as a nonselectable, stable, high copy number yeast vector. Plasmid. 1991;25(2):81-95.

https://doi.org/10.1016/0147-619X(91)90019-S

48. Karim AS, Curran KA, Alper HS. Characterization of plasmid burden and copy number in Saccharomyces cerevisiae for optimization of metabolic engineering applications. FEMS Yeast Res. 2013;13(1):107-16. https://doi.org/10.1111/1567-1364.12016

49. Wu A, Wemmie JA, Edgington NP, Goebl M, Guevara JL, Moye-Rowley WS. Yeast bZip proteins mediate pleiotropic drug and metal resistance. J Biol Chem. 1993;268(25):18850-8.

50. Stephen DWS, Rivers SL, Jamieson DJ. The role of the YAP1 and YAP2 genes in the regulation of the adaptive oxidative stress responses of Saccharomyces cerevisiae. Mol Microbiol. 1995;16(3):415-23.

https://doi.org/10.1111/j.1365-2958.1995.tb02407.x

51. Kuge $\mathrm{S}$, Jones N, Nomoto, A. Regulation of yAP-1 nuclear localization in response to oxidative stress. EMBO J. 1997;16(7):1710-20. https://doi.org/10.1093/emboj/16.7.1710

52. Okazaki S, Tachibana T, Naganuma A, Mano N, Kuge S. Multistep disulfide bond formation in Yap1 is required for sensing and transduction of $\mathrm{H}_{2} \mathrm{O}_{2}$ stress signal. Mol Cell. 2007;27(4):675-88.

https://doi.org/10.1016/j.molcel.2007.06.035

53. Kanazawa S, Driscoll M, Struhl K. ATR1, a Saccharomyces cerevisiae gene encoding a transmembrane protein required for aminotriazole resistance. Mol Cell Biol. 1988;8(2):664-73. https://doi.org/10.1128/MCB.8.2.664 